\title{
Tyr78Phe Transthyretin Mutation with Predominant Motor Neuropathy as the Initial Presentation
}

\author{
Giulietta Riboldi Roberto Del Bo Michela Ranieri \\ Francesca Magri Monica Sciacco Maurizio Moggio \\ Nereo Bresolin Stefania Corti Giacomo P. Comi \\ Dino Ferrari Centre, Department of Neurological Sciences, University of Milan, \\ IRCCS Foundation Ca' Granda, Ospedale Maggiore Policlinico, Milan, Italy
}

\section{Key Words}

Amyloid neuropathy - Motor-sensory neuropathy · Transthyretin gene

\begin{abstract}
Transthyretin (TTR) amyloidosis, the most frequent form of hereditary amyloidosis, is caused by dominant mutations in the TTR gene. More than 100 mutations have been identified. Clinical manifestations of TTR amyloidosis are usually induced by extracellular amyloid deposition in several organs. The major neurological manifestation is motorsensory neuropathy associated with dysautonomic impairment. Here, we describe a 63-year-old man who came to our institution due to a suspected motor neuron disease. During a 4-year follow-up period, he underwent extensive clinical examination, electromyographic studies, sural nerve biopsy and TTR gene analysis by direct sequencing. Despite the predominant motor involvement, the detailed clinical examination also showed some mild sensory and dysautonomic signs. In addition, his clinical and family history included multiorgan disorders, such as carpal tunnel syndrome, as well as conditions with cardiac, renal, eye, and hepatic involvement. The sural nerve biopsy disclosed amyloid deposition, and the sequence analysis of the TTR gene detected a heterozygous Tyr78Phe substitution. The TTR gene variant found in our patient had only been described once so far, in a French man of Italian origin presenting with late-onset peripheral neuropathy and bilateral carpal tunnel syndrome. The predominant motor involvement presented by our patient is an uncommon occurrence and demonstrates the clinical heterogeneity of TTR amyloidosis.
\end{abstract}




\section{Introduction}

Hereditary amyloidosis represents a clinically and genetically heterogeneous group of autosomal dominant inherited diseases characterized by the deposit of insoluble variants of physiological proteins in the extracellular matrix [1]. Mutated transthyretin (TTR), formerly called prealbumin and encoded by a gene on chromosome 18, is the most frequent protein implicated, whereas apolipoprotein A1, lysozyme, fibrinogen, gelsolin, amyloid- $\beta$ and cystatin $C$ are much more uncommon [2]. TTR is a tetrameric serum protein involved in the transport of serum thyroxine and retinol-binding protein synthesized in the liver and, minimally, in the choroid plexus. Its variation enables the dissociation of tetrameric TTR, which increases the concentration of aberrant amyloidogenic monomers. TTR amyloidosis is characterized by slowly progressive peripheral sensorimotor and autonomic neuropathy $[1,3]$ as well as cardiomyopathy [4], nephropathy, vitreous opacities, and central nervous system involvement [5]. Portugal and Japan are the countries with the largest endemic foci [6].

In this study, we report a 63-year-old man who carries a missense mutation in the TTR gene and presented with rapidly progressive axonal motor neuropathy, clinically resembling a motor neuron disease, with subclinical signs of sensory involvement.

\section{Case Report}

The patient first came to our attention when he was 63 years old. He presented with increasing weakness of the lower limbs, muscle fatigue, and dysphagia, which began at the age of 59 years. His neurological symptoms worsened over the years, resulting in a greater difficulty in climbing the stairs and standing up from a chair.

He had a positive cardiological history that included supraventricular arrhythmia (treated with ablation of the arrythmogenic focus) and atrial fibrillation (which resolved after pharmacological therapy). In addition, he had a right eye cataract, Paget's disease, chronic renal failure due to glomerulonephritis, gastroesophageal reflux, and hepatic steatosis. At 45 and 55 years of age, the patient underwent surgical decompressions for bilateral carpal tunnel syndrome with a secondary lesion of the right median nerve. His family history was positive for cardiomyopathy, type II diabetes mellitus, hypertension, cancer, and Parkinson's disease. Neuromuscular disorders were not evident in the family history. The patient's father died at 63 years of age due to renal failure, and his mother died at 69 years of age due to ovarian neoplasm. He had 1 brother and 1 sister, both of whom had bilateral carpal tunnel syndrome and myopia; the brother also had cataracts and the sister underwent surgery for uterine neoplasm.

The patient was admitted to another hospital, where he underwent several investigations. An electromyogram (EMG) detected an axonal motor neuropathy with minimal sensory impairment; the amplitude of the sensory nerve action potential was decreased ( $4 \mu \mathrm{V}$ right; $6 \mu \mathrm{V}$ left). A muscle biopsy, performed on the quadriceps femoris, showed a neurogenic pattern characterized by high size variability, with several atrophic fibers and fiber type II grouping. Connective tissue increase, cellular infiltrates as well as necrotic fibers were absent. A brain MRI and cerebrospinal fluid analysis showed normal results. Anti-GM1 IgM antibodies were mildly increased (1/640).

In the following months, the patient exhibited a severe and rapid progression of the motor impairment of the 4 limbs, characterized by muscle atrophy and fasciculation, without comparable clinical defect of the sensory component. Since the subclinical sensory impairment, only detectable with electrophysiology, was interpreted as incidental finding, the patient was diagnosed with probable amyotrophic lateral sclerosis (ALS), although his clinical presentation was more suggestive of progressive muscular atrophy; he was therefore referred to our motor neuron disease center. 
The patient underwent an extensive clinical assessment, comprehensive of family and personal history data recording, complete neurological examination, and muscle strength evaluation using the Medical Research Council and ALS Functional Rating Scale.

Our neurological examination revealed bulbar signs such as tongue atrophy and fasciculation, nasal voice, and dysarthria. The strength of the trunk and upper proximal limb muscles was normal. The patient presented with some difficulty in digital skills, hand weakness with diffuse fasciculation, and atrophy of the right arm. He had bilateral mild proximal muscle weakness in the lower limbs, with a particular atrophy in the distal regions (right $>$ left). He was able to walk on his own, albeit on tiptoe. Ubiquitous absence of osteotendon reflexes was detected. A sensory deficit was observed only in the first 3 fingers of the right hand. His ALS Functional Rating Scale score was 31.

The EMG was repeated and showed a pattern characterized by electroneurographical findings compatible with motor-sensory neuropathic involvement. At nerve conduction study, the tibial posterior nerve showed a reduced compound muscle action potential amplitude $(0.9$ and $0.5 \mathrm{mV})$ and a reduced nerve conduction velocity (NCV; 36.5 and $36.4 \mathrm{~m} / \mathrm{s}$ ). The sensitive NCV of the sural nerve was reduced bilaterally $(36.2$ and $33.3 \mathrm{~m} / \mathrm{s})$, as were the NCV and sensitive action potential amplitude of the ulnar $(46.6 \mathrm{~m} / \mathrm{s})$ and radial $(50 \mathrm{~m} / \mathrm{s})$ nerves. The concentric electric needle examination demonstrated neurogenic signs predominantly at the lower limbs, with fibrillation potentials and motor unit action potentials characterized by prolonged amplitude and duration. A paraspinal muscle EMG was also performed and revealed the presence of fibrillation.

Since the patient had a sporadic occupational exposure to polychlorinated biphenyls, he was also examined by a specialist in occupational medicine who excluded any possible link between the patient's polyneuropathy and work. Other genetic causes of motor neuron syndromes were also excluded. The superoxide dismutase 1 gene was screened as the first gene in our familial ALS assessment protocol, and the valosin-containing protein gene was analyzed because of the comorbid Paget's disease. Both analyses showed negative results.

A more detailed medical history investigation revealed the presence of dysautonomia presenting as erectile dysfunction that was not previously reported. Neither the patient's history nor clinical examination revealed any other dysautonomic symptoms. For this reason, no additional autonomic tests were performed, but specific tests to examine the sensory and dysautonomic involvement will be considered during follow-up. The remote and familial history and the findings of dysautonomia and mild sensory impairment associated with systemic aspects (above-mentioned cardiac, ocular and renal features) suggested revising the patient's diagnosis, and amyloid etiopathogenesis was considered.

A periumbilical fat biopsy was performed and showed no amyloid deposition. We decided to perform a TTR gene molecular analysis and detected the presence of a heterozygous missense mutation (p.Tyr78Phe; c.293A>T according to Ensembl genome browser ID OTTHUMT00000254948) (fig. 1). Samples from the patient's parents were not available for further investigation because both were deceased and other siblings were not available for analysis. The pathogenic role of this mutation was confirmed through a sural nerve biopsy analysis, which showed a mild axonal neuropathic pattern characteristic of amyloidosis and demonstrated a reduced myelinization that was more evident in semithin sections, with conserved bimodal distribution. Endoneurial connective tissue was increased, blood vessels were normal, and cellular infiltrates were absent. The quantitative analysis showed a fiber density of 1,475 nerve fibers $/ \mathrm{mm}^{2}$ (normal value according to the age: $4,800 \pm 1,000$ ). A teasing technique showed a normal aspect in $49 \%$ of the fibers, total demyelinization in $41 \%$, and axonal degeneration in $6 \% ; 2 \%$ presented with segmental demyelinization or remyelinization, respectively (舀. 2 ).

The disease was confirmed through specific staining with Congo red, which revealed strongly positive deposits in several capillary walls that appeared as apple-green birefringence when examined under polarized light.

Given that liver transplantation is still the only recognized therapy for TTR polyneuropathy, this possibility was proposed to our patient, who is now waiting for transplantation. 


\begin{tabular}{l|l|l|l} 
Case Reports in & $\begin{array}{l}\text { Case Rep Neurol 2011;3:62-68 } \\
\text { DOl: } 10.1159 / 000324925\end{array}$ & $\begin{array}{l}\text { Published online: } \\
\text { February 23, 2011 }\end{array}$ & $\begin{array}{l}\text { ISSN 1662-680X } \\
\text { ISww.karger.com/crn }\end{array}$ \\
\hline
\end{tabular}

\section{Discussion}

Mutations in the TTR gene are among the most frequent causes of hereditary autosomal dominant amyloidosis and are mainly responsible for familial amyloid polyneuropathy (FAP), which is characterized by predominant sensorimotor and autonomic involvement due to endoneurial and polyvisceral extracellular deposition of amyloid substance [7]. Interestingly, motor function is preserved until the sensory neuropathy has advanced considerably.

Here, we describe the case of a patient with predominant motor involvement, leading to a diagnostic hypothesis of motor neuron disease. The major clinical features of this patient were distal limb paralysis, atrophy, fasciculations, dysphagia, and dysarthria. The sensory and autonomic deficits were minimal and were only identified after accurate electroneurographic study and complete clinical data collection. These aspects, in association with the patient's past medical history of carpal tunnel syndrome as well as the presence of some systemic defects such as renal impairment, cardiac fibrillation, and cataracts, led to the suspicion of amyloidosis.

Molecular analysis revealed the presence of the heterozygous missense mutation p.Tyr78Phe in the TTR gene. The pathogenicity of this mutation and the link between amyloid deposition and polyneuropathy were confirmed histologically by the sural nerve biopsy, which demonstrated mild peripheral axonal neuron sufferance with positive birefringent deposits at Congo red staining. The amyloidogenic properties of the Tyr78Phe mutation of the TTR gene have also been demonstrated by Redondo et al [8], who showed that the substitution of residue 78 led to a changed tetrameric structure that might represent an early intermediate in the fibrillogenesis pathway.

Although the p.Tyr78Phe mutation is rare, it has been described once before in a 78-year-old French man of Italian origin who was affected with late-onset peripheral neuropathy in the lower limbs, bilateral carpal tunnel, and skin amyloidosis [9]. More than 100 pathogenic mutations leading to misfolding and formation of amyloid deposits have been detected in the TTR gene [10]. These mutations are mainly single nucleotide substitutions with an autosomal pattern of transmission but variable penetrance [11]. Among them, the substitution of a methionine at codon 30 (p.Val30Met) was the first to be described [12] and so far remains the most frequent, especially in Portugal, North Sweden, and Japan. Some mutations are associated with a specific clinical presentation, such as predominant cardiomyopathy [13]. Other variants can present a wide clinical interfamilial variability [14], probably due to epigenetic and environmental factors. Usually, FAP is progressing faster than in our patient, and even if there are no studies to date correlating TTR mutations and survival, the very rare mutation in our patient could be related to a disease form with longer evolution.

The peculiar aspect in this case was the unusual manifestation of polyneuropathy with a predominant motor involvement and only minimal and late-onset sensory and dysautonomic signs. Sensory and autonomic involvement can be subtly but definitely present in amyloidosis, and new investigation tools may help to test sensitive and dysautonomic symptoms in TTR mutation polyneuropathy and to identify the amyloid pathologic substrate. The value of standard quantitative autonomic and sensation tests in detecting, characterizing, and quantifying the severity of TTR amyloid polyneuropathy has recently been assessed [15]. In addition, the quantitative assessment of cutaneous 
nerve fibers can be employed as an indicator of the severity of neuropathy [16]. Identifying a specific causative amyloid protein from nerves and other tissues is often difficult but important in diagnosis and treatment. The possibility of distinguishing the specific type of amyloidosis from nerve biopsies combining laser microdissection and mass spectrometric-based proteomic analysis has also been recently described. This new proteomic approach can promote both diagnostic and research efforts in amyloidosis [17]. Some of these methods are still experimental and were not applied in our case, but they may be considered for the assessment and follow-up of TTR patients.

The above-mentioned atypical manifestation of FAP has only been described in a single case by Quattrini et al. [18]. Because of this finding, amyloid polyneuropathy should be considered as an alternative diagnosis of motor neuron disease, especially in the presence of systemic symptoms, such as carpal tunnel syndrome and cataract, renal, heart, or skin involvement. The clinical phenotypic heterogeneity of TTR mutations and the predominant motor or sensory/dysautonomic pattern can be explained by the complex multifactorial mechanisms that regulate amyloid fibrillogenesis in FAP, such as the intrinsic conformation of the TTR fibril protein, its concentration, the structural effects of the TTR mutation, microenvironments of the lysosome, proteolytic processing, amyloid cofactors, and tissue-specific determinants [19].

We underscore that the negative results from the periumbilical fat and muscle biopsies does not exclude the diagnosis. In fact, in our patient the periumbilical fat biopsy did not reveal amyloid deposition, and the muscle biopsy must be completed with specific methods, such as Congo red staining, which are not routinely used.

The recognition of amyloid etiologies in patients affected with predominant motor polyneuropathy with few sensory signs is fundamental. In fact, the molecular definition of this disease can help in genetic counseling, because the mutation is inherited in an autosomal dominant manner [6].

Furthermore, the early diagnosis of amyloid neuropathy allows the timely starting of potential therapies, such as liver transplantation.

\section{Acknowledgements}

We wish to thank the patients and their families for their support and collaboration. This research received funding support from Telethon - UILDM Grant GUP08005 (GPC). Telethon Genetic Biobanks Network GTB07001E provided the DNA used in this study. Eurobiobank project QLTR-200102769 is also gratefully acknowledged.

\section{Disclosure Statement}

The authors have no conflicts of interest to declare. 


\begin{tabular}{l|l|l|l}
$\begin{array}{c}\text { Case Reports in } \\
\text { NeUlology }\end{array}$ & $\begin{array}{l}\text { Case Rep Neurol 2011;3:62-68 } \\
\text { DOI: 10.1159/000324925 }\end{array}$ & $\begin{array}{l}\text { Published online: } \\
\text { February 23, 2011 }\end{array}$ & $\begin{array}{l}\text { O 2011 S. Karger AG, Basel } \\
\text { ISSN 1662-680X } \\
\text { www.karger.com/crn }\end{array}$ \\
\hline
\end{tabular}

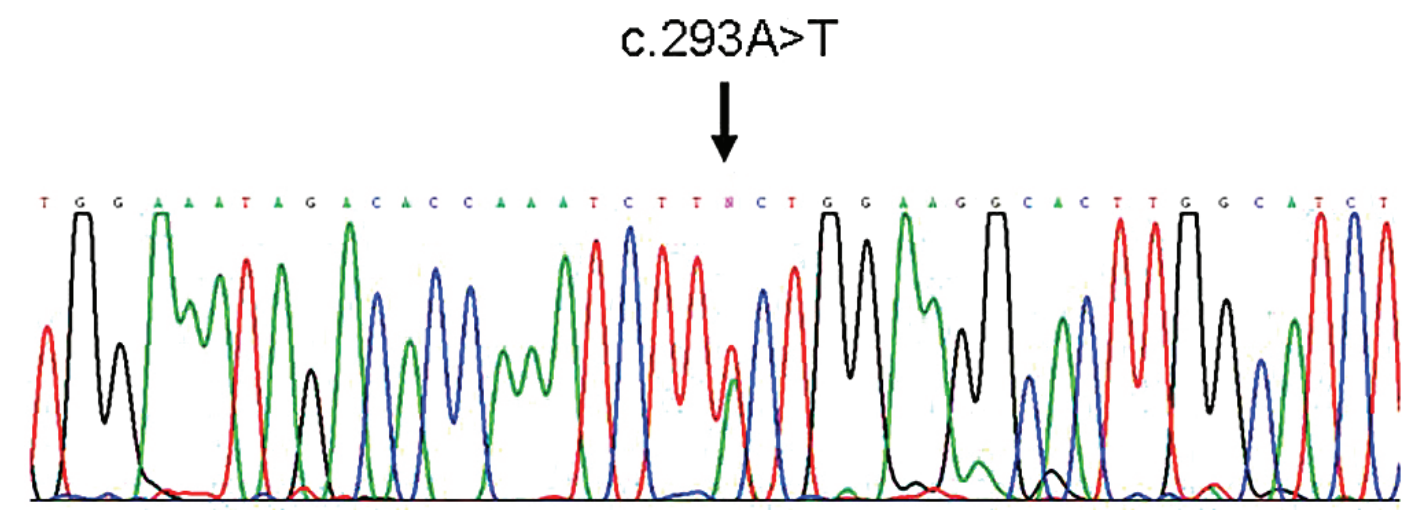

Fig. 1. Sequence analysis showing the nucleotide substitution c.10783C $>\mathrm{T}$, which leads to the heterozygous missense mutation Tyr78Phe in exon 1.
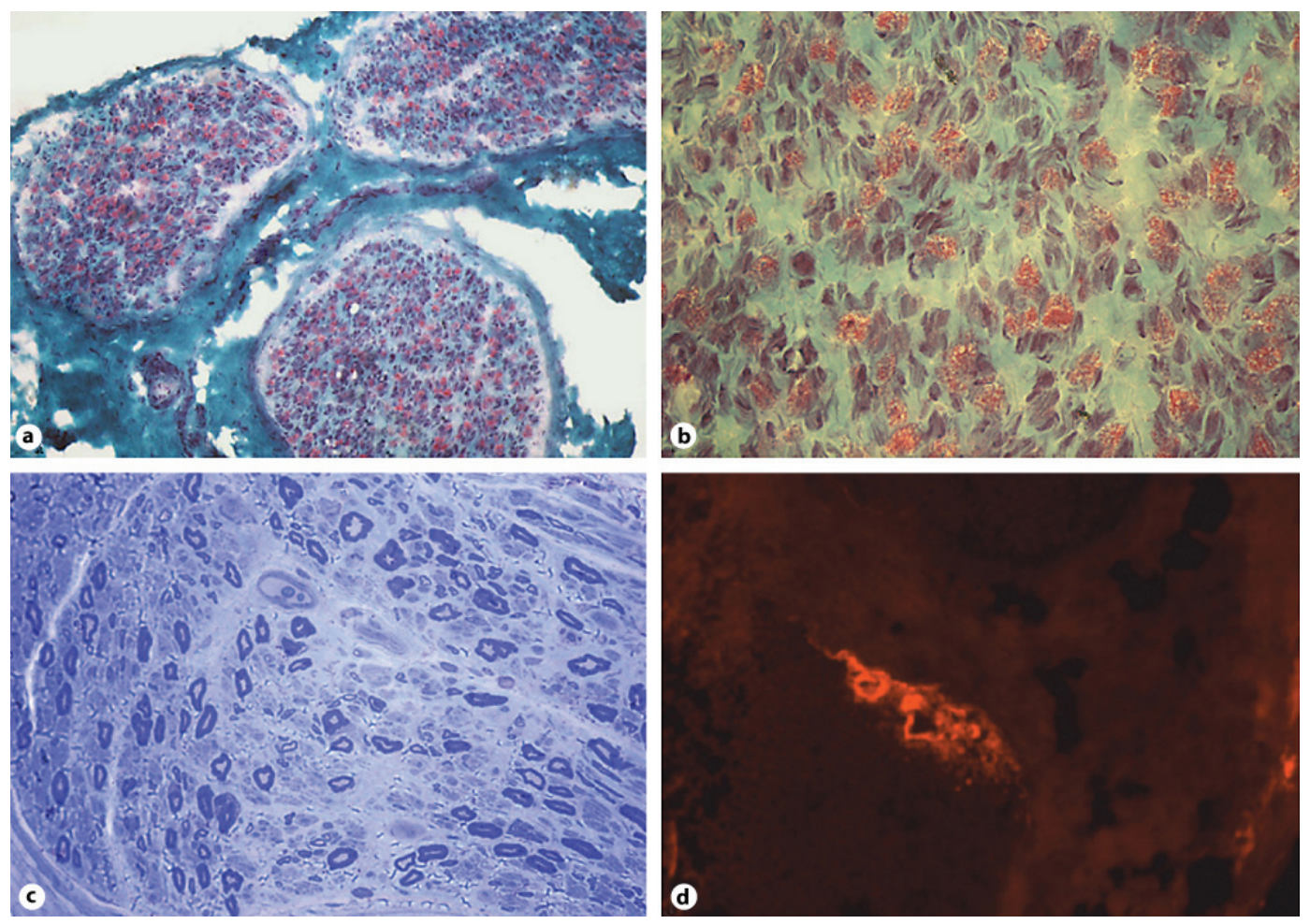

Fig. 2. a, b Light microscopy: Gomori's trichrome stain of 3 nerve fascicles shows moderate to severe loss of myelinated fibers $(\mathbf{a}, \times 100 ; \mathbf{b}, \times 400)$. c Semithin cross-section confirms severe reduction of myelinated fiber density (toluidine blue stain, $\times 400$ ). $\mathbf{d}$ Deposition of amyloid identified by Congo red $(\times 200)$. 


\begin{tabular}{c|l|l|l} 
Case Reports in & Case Rep Neurol 2011;3:62-68 & Published online: & $\begin{array}{l}\odot \text { 2011 S. Karger AG, Basel } \\
\text { ISSN 1662-680X } \\
\text { www.karger.com/crn }\end{array}$ \\
\hline
\end{tabular}

\section{References}

1 Hund E, Linke RP, Willig F, Grau A: Transthyretin-associated neuropathic amyloidosis. Pathogenesis and treatment. Neurology 2001;56:431-435.

2 Benson DA, Karsch-Mizrachi I, Lipman DJ, Ostell J, Wheeler DL: GenBank: update. Nucleic Acids Res 2004;32:D23-D26.

-3 Ando Y, Nakamura M, Araki S: Transthyretin-related familial amyloidotic polyneuropathy. Arch Neurol 2005;62:1057-1062.

4 Nakazato M: Genotype-phenotype relationship in familial amyloid polyneuropathy. Neurol Med (Tokyo) 1998;48:528-534.

5 Benson MD: Ostertag revisited: the inherited systemic amyloidoses without neuropathy. Amyloid 2005;12:7587.

6 Sekijima Y, Yoshida K, Tokuda T, Ikeda S: Familial transthyretin amyloidosis; in Pagon RA, Bird TC, Dolan CR, Stephens K (eds): GeneReviews. Seattle, University of Washington, 1993.

7 Planté-Bordeneuve V, Said G: Transthyretin related familial amyloid polyneuropathy. Curr Opin Neurol 2000;13:569-573.

-8 Redondo C, Damas AM, Olofsson A, Lundgren E, Saraiva MJ: Search for intermediate structures in transthyretin fibrillogenesis: soluble tetrameric Tyr78Phe TTR expresses a specific epitope present only in amyloid fibrils. J Mol Biol 2000;304:461-470.

-9 Magy N, Liepnieks JJ, Gil H, Kantelip B, Dupond JL, Kluve-Beckerman B, Benson MD: A transthyretin mutation (Tyr78Phe) associated with peripheral neuropathy, carpal tunnel syndrome and skin amyloidosis. Amyloid 2003;10:29-33.

10 Connors LH, Lim A, Prokaeva T, Roskens VA, Costello CE: Tabulation of human transthyretin (TTR) variants. Amyloid 2003;10:160-184.

11 Coelho T, Sousa A, Lourenço E, Ramalheira J: A study of 159 Portuguese patients with familial amyloidotic polyneuropathy (FAP) whose parents were both unaffected. J Med Genet 1994;31:293-299.

12 Tashima K, Ando Y, Ando E: Heterogeneity of clinical symptoms in patients with familial amyloidotic polyneuropathy (FAP TTR Met30). Amyloid 1997;4:108-111.

13 Rosenzweig M, Skinner M, Prokaeva T, Théberge R, Costello C, Drachman BM, Connors LH: A new transthyretin variant (Glu61Gly) associated with cardiomyopathy. Amyloid 2007;14:65-71.

14 Noto Y, Tokuda T, Shiga K, Tsuchiya A, Yazaki M, Matoba S, Nakagawa M: Cardiomyopathy in a Japanese family with the Glu61Lys transthyretin variant: a new phenotype. Amyloid 2009;16:99-102.

15 Kim DH, Zeldenrust SR, Low PA, Dyck PJ: Quantitative sensation and autonomic test abnormalities in transthyretin amyloidosis polyneuropathy. Muscle Nerve 2009;40:363-370.

-16 Yang NC, Lee MJ, Chao CC, Chuang YT, Lin WM, Chang MF, Hsieh PC, Kan HW, Lin YH, Yang CC, Chiu MJ, Liou HH, Hsieh ST: Clinical presentations and skin denervation in amyloid neuropathy due to transthyretin Ala97Ser. Neurology 2010;75:532-538.

17 Klein CJ, Vrana JA, Theis JD, Dyck PJ, Dyck PJ, Spinner RJ, Mauermann ML, Bergen HR 3rd, Zeldenrust SR, Dogan A: Mass spectrometric-based proteomic analysis of amyloid neuropathy type in nerve tissue. Arch Neurol 2010, E-pub ahead of print.

18 Quattrini A, Nemni R, Sferrazza B, Ricevuti G, Dell'Antonio G, Lazzerini A, Iannaccone S: Amyloid neuropathy simulating lower motor neuron disease. Neurology 1998;51:600-602.

19 Herbert J: Familial amyloidotic polyneuropathy; in Rosenberg RN, Prusiner SB, DiMauro S, Barchi RL (eds): The Molecular and Genetic Basis of Neurological Disease. Boston, Butterworth-Heinemann, 1997, pp 845-864. 